\title{
Research on Measurement of Resource Consumption Based on Time Correlation
}

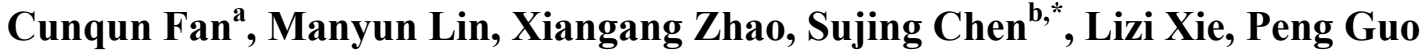 \\ National Satellite Meteorological Centre, Beijing, China \\ a email:fancq@cma.gov.cn, ${ }^{\mathrm{b}}$ email:chensj@cma.gov.cn \\ *corresponding author
}

Keywords: Meteorological satellite; Ground application system; Resource consumption; Time correlation

\begin{abstract}
Meteorological satellite terrestrial applications bear a large number of applications, these applications correspond to a variety of processing tasks, and these tasks have different consumption of resources, how to properly measure the consumption of these resources is to improve the overall efficiency of the system key. Aiming at this problem, this paper proposes a resource consumption measurement method based on time correlation. First of all, the variance and covariance of resources and time are calculated, then the correlation between resources and time is obtained, and then the impact of time on resource consumption is judged.
\end{abstract}

\section{Introduction}

The applications of meteorological satellite ground applications are large and complex, and the requirements of different types of applications are quite different from those of resources. Reasonable measurement of the consumption of these resources by the meteorological satellite ground application system is the key to improve the overall operation efficiency of the system. How to find out the relationship between system resource consumption and other parameters, and then measure the system resource consumption has become a key issue.

Some related achievements have been made on the related research on resource measurement. The paper [1] formalized a general problem of analyzing resource usage as a resource usage analysis problem, and proposed a type-based method as a solution to the problem. The paper [2] provided analysis of resource usage and requirements and is an attempt to give an insight into such kind of production trace similar to the ones in cloud environment. The major contributions of the paper include Statistical Profile of Jobs based on resource usage, clustering of Workload Patterns and Classification of jobs into different types based on k-means clustering. In paper [3], design of an Android-based augmented reality application is presented and particularly its performance is analyzed in terms of resource usage in comparison to similar applications. The application displays merchant, branch information of one of the Turkish banks, as well as related sales campaigns of the merchants on the screen that are within the proximity of the user's location. The paper [4] proposed a methodology for measuring and modeling the performance of hierarchical memories in terms of 
the application's utilization of the key memory resources: capacity of a given memory level and bandwidth between two levels. This is done by actively interfering with the application's use of these resources. The application's sensitivity to reduced resource availability is measured by observing the effect of interference on application performance. The paper [5] proposed an optimistic adaptive monitoring system to determine the faulty components of an application. Suspected components are finely analyzed by the monitoring system, but only when required. Unsuspected components are left untouched and execute normally.

Based on the above research, this paper proposes a resource consumption measurement method based on time correlation. First of all, the variance and covariance of resources and time are calculated, then the correlation between resources and time is obtained, and then the impact of time on resource consumption is judged.

\section{The Definition of Time-related}

The purpose of time-related analysis is to explore the potential linkages between application resource consumption and overall time running time, and to assess the impact of different application resource consumption on the overall application running time. Evaluate the profile characteristics of application runtime affected by resource consumption and plot the application runtime dependencies so that you can draw significant metrics that impact application runtime.

Time-related analysis Pearson's correlation coefficient was calculated by analyzing the degree of depletion of different resources exhibited by the application during multiple runs and the length of running time. The coefficients obtained are used to describe the impact of resource consumption on application runtime. The high correlation indicates that the resource consumption has a great influence on the operation time, and the low correlation means that the impact of resource consumption on the operation time is low.

Simple correlation coefficient classification is as follows.

- 0.8-1.0 are strongly related.

- 0.6-0.8 are moderately strong correlation.

- 0.4-0.6 are moderately relevant.

- 0.2-0.4 are weakly related.

- 0.0-0.2 are very weak or no correlation.

\section{Time Correlation Algorithm}

Define a job In the $i$-th scheduling process, the load average of resource $j$ is $x_{i, j}$, the running time of the scheduling is $t_{i}$, assuming that the job has been dispatched a total of $n$ times, there are $m$ different kinds of resources.

The average of resource $i$ scheduling in $n$ times is defined as follows.

$$
\bar{X}_{j}=\sum_{i=1}^{\Pi} X_{i, j}
$$

The average time consumption of secondary scheduling is:

$$
\bar{t}=\sum_{i=1}^{n} t_{i}
$$

The variance between resource $x_{i}$ and time $t$ is:

$$
D\left(x_{j}\right)=\frac{1}{n} \sum_{i=1}^{n}\left(x_{i j}-\bar{x}_{i}\right)\left(x_{i j}-\bar{x}_{j}\right)
$$




$$
D(t)=\frac{1}{n} \sum_{i=1}^{n}\left(t_{i}-\bar{t}\right)^{2}
$$

The covariance between resource $\mathrm{N}$ and time $\mathrm{T}$ is defined as follows.

$$
\operatorname{Cov}\left(x_{j}, t\right)=\frac{1}{n} \sum_{i=1}^{n}\left(x_{i j}-\bar{x}_{j}\right)\left(t_{i}-\bar{t}\right)
$$

In order to avoid the situation where the divisor is 0 and $\varepsilon$ is set to a very small number, the correlation coefficient between the resource $x_{i}$ and the time $t$ is defined as follows.

$$
\rho_{j}=\frac{\operatorname{cov}\left(x_{j}, t\right)}{\sqrt{D\left(x_{j}\right) D(t)}+\varepsilon}
$$

\section{The Concrete Realization of Time Relevance}

The relevance of time-related value range, the relevance of the actual application are generally relatively low. This is because there are many factors that affect the application run time, and the resulting correlation with a certain resource consumption will not be too high.

Relevant scores appear 0 situation may have the following two kinds:

(1) A job runs only once in the job stream.

(2) 2 jobs in a number of scheduling resource consumption or running time has not changed.

The more times a job is scheduled, the higher the credibility of its score. The appearance of Case 1 shows that the result of this correlation analysis is not credible.

\section{Conclusions}

Meteorological satellite terrestrial applications bear a large number of applications, these applications correspond to a variety of processing tasks, and these tasks have different consumption of resources, how to properly measure the consumption of these resources is to improve the overall efficiency of the system key. Aiming at this problem, this paper proposes a resource consumption measurement method based on time correlation. First of all, the variance and covariance of resources and time are calculated, then the correlation between resources and time is obtained, and then the impact of time on resource consumption is judged.

\section{Acknowledgements}

The work presented in this study is supported by National High-tech R\&D Program (2011AA12A104).

\section{References}

[1] Igarashi A, Kobayashi N. Resource usage analysis[J]. Acm Sigplan Notices, 2005, 27(2):264-313.

[2] Alam M, Shakil K A, Sethi S. Analysis and Clustering of Workload in Google Cluster Trace based on Resource Usage[J]. Computer Science, 2015.

[3] Karaman A, Erisik D, Incel O D, et al. Resource Usage Analysis of a Sensor-based Mobile Augmented Reality Application is [J]. Procedia Computer Science, 2016, 83:300-304.

[4] Casas M, Bronevetsky G. Evaluation of HPC Applications' Memory Resource Consumption via Active Measurement[J]. IEEE Transactions on Parallel \& Distributed Systems, 2016, 27(9):2560-2573.

[5] Gonzalez-Herrera I, Bourcier J, Daubert E, et al. ScapeGoat: Spotting abnormal resource usage in componentbased reconfigurable software systems[J]. Journal of Systems \& Software, 2016, 122:398-415. 